\title{
Chain Multiplication of Fatty Acids to Precise Telechelic Polyethylene
}

\author{
Timo Witt ${ }^{+}$, Manuel Häußler ${ }^{+}$, Stefanie Kulpa, and Stefan Mecking*
}

\begin{abstract}
Starting from common monounsaturated fatty acids, a strategy is revealed that provides ultra long aliphatic $\alpha, \omega$ difunctional building blocks by a sequence of two scalable catalytic steps that virtually double the chain length of the starting materials. The central double bond of the $\alpha, \omega$ dicarboxylic fatty acid self metathesis products is shifted selectively to the statistically much disfavored $\alpha, \beta$ position in a catalytic dynamic isomerizing crystallization approach. "Chain doubling” by a subsequent catalytic olefin metathesis step, which overcomes the low reactivity of this substrates by using waste internal olefins as recyclable co reagents, yields ultra long chain $\alpha, \omega$ difunctional building blocks of a precise chain length, as demonstrated up to a $C_{48}$ chain. The unique nature of these structures is reflected by unrivaled melting points $\left(T_{m}=120^{\circ} \mathrm{C}\right)$ of aliphatic polyesters generated from these telechelic monomers, and by their self assembly to polyethylene like single crystals.
\end{abstract}

O wing to their particular molecular structure, naturally occurring fatty acids are a feedstock of choice for the generation of molecules comprising long methylene sequen ces with an attached functional group. The generation of sebacic acid from castor oil is a well established example. This mid chain dicarboxylic acid is used as a monomer building block for high performance nylons ${ }^{[1]}$ or biodegradable poly esters. ${ }^{[2]}$ Recently developed catalytic routes allow for the preparation of other long chain $\alpha, \omega$ difunctional compounds from readily available fatty acids, such that the product comprises the full length of a fatty acid chain. For example, dicarboxylic acids HOOC $\left(\mathrm{CH}_{2}\right)_{n} \mathrm{COOH}$ with $n=18$ or 19 can be generated from the $\mathrm{C}_{18}$ fatty acids oleic or stearic acid. ${ }^{[3,4]}$

Beyond these chain lengths, ultra long chain compounds with methylene sequences that significantly exceed the length of a typical fatty acid chain appear relevant from different perspectives. For instance, such structural motifs occur as ceramide, ${ }^{[5,6]}$ a main component of the stratum corneum of the epidermis layer of the human skin, and they are part of the cellular walls of several algae species (algaenans) ${ }^{[711]}$ and of plants (cutin and suberin)..$\left.^{12} 14\right]$ The highly hydrophobic nature of the $\alpha, \omega$ difunctional building blocks here is exploited to generate an insoluble polymer, protecting the

[*] T. Witt, ${ }^{[+]}$M. Häußler, ${ }^{[+]}$S. Kulpa, Prof. Dr. S. Mecking

Chair of Chemical Materials Science, Department of Chemistry,

University of Konstanz

Universitätsstraße 10, 78457 Konstanz (Germany)

E mail: stefan.mecking@uni konstanz.de

$\left.{ }^{+}\right]$These authors contributed equally to this work. organisms from undesired influences from their environment as well as from water loss. From a different viewpoint, their length corresponds to the typical thickness of phospholipid bilayers, which offers possibilities for synthetic structures mimicking these ubiquitous structures of biological sys tems. ${ }^{[15]}$ Further, such chain lengths are also found as the typical thickness of crystalline lamellae in polymers, which determines their materials properties. This suggests that there are specific lengths of a methylene sequence, which show an extraordinary strong driving force for crystallization. Artifi cial ultra long chain compounds with these lengths thus might be powerful building blocks for various crystalline materials.

However, the very limited perspectives for accessing such compounds efficiently has discouraged broader studies of their properties and potential. Reported strategies for the synthesis of aliphatic $\alpha, \omega$ difunctional building blocks with a chain length greater than $\mathrm{C}_{30}$ commonly comprise tedious multi step reaction sequences. For example, multiple cycles of Wurtz coupling of $\alpha$ halo $\omega$ alcohols can be used for the preparation of a $\mathrm{C}_{44}$ diol. ${ }^{[16]}$ Catalytic methods have also been investigated, as reported by Augustin and Schäfer in a Glaser coupling starting from oleic acid. ${ }^{[17]}$ Yet again, laborious steps for the preparation of a terminal acetylene are required to access the desired bis(triunsaturated) $\mathrm{C}_{36} \alpha, \omega$ diester. Addi tionally, the reagents and by products involved in all these procedures are prohibitive to performing them on larger scales. As an example of entirely different polymerization approaches, catalyzed chain growth of ethylene can yield difunctional chains. Limitations of these approaches are imprecise chain lengths and imperfect end group fidelity. Additionally, in most cases specific functionalized magnesium reagents and additional post polymerization steps are required that are not well suited for large scales. $\left.{ }^{[18} 20\right]$

We now report an approach that allows for the doubling of the chain length of readily available fatty acid starting materials, employing catalyzed steps exclusively which also do not consume further reagents and do not generate excessive amounts of undesired side products. With this technique, $\alpha, \omega$ difunctional building blocks with a $\mathrm{C}_{48}$ chain can be accessed that open opportunities as exemplified by self assembled structures and crystalline materials.

Self metathesis of unsaturated fatty acid derivatives, as a well established and industrially used process, ${ }^{[21]}$ allows the synthesis of internally unsaturated dicarboxylic compounds. These reactions, generally leading to equilibrium mixtures of products and starting material, can be driven in a favorable direction by choosing appropriate reaction conditions. ${ }^{[22,23]}$

By application of Hoveyda Grubbs second generation complex (Cat1) as a metathesis catalyst, the reaction equilib rium of oleic acid (1a) can be driven to the diacid (and stoichiometric amounts of octadecene) by its precipitation from the reaction mixture. 


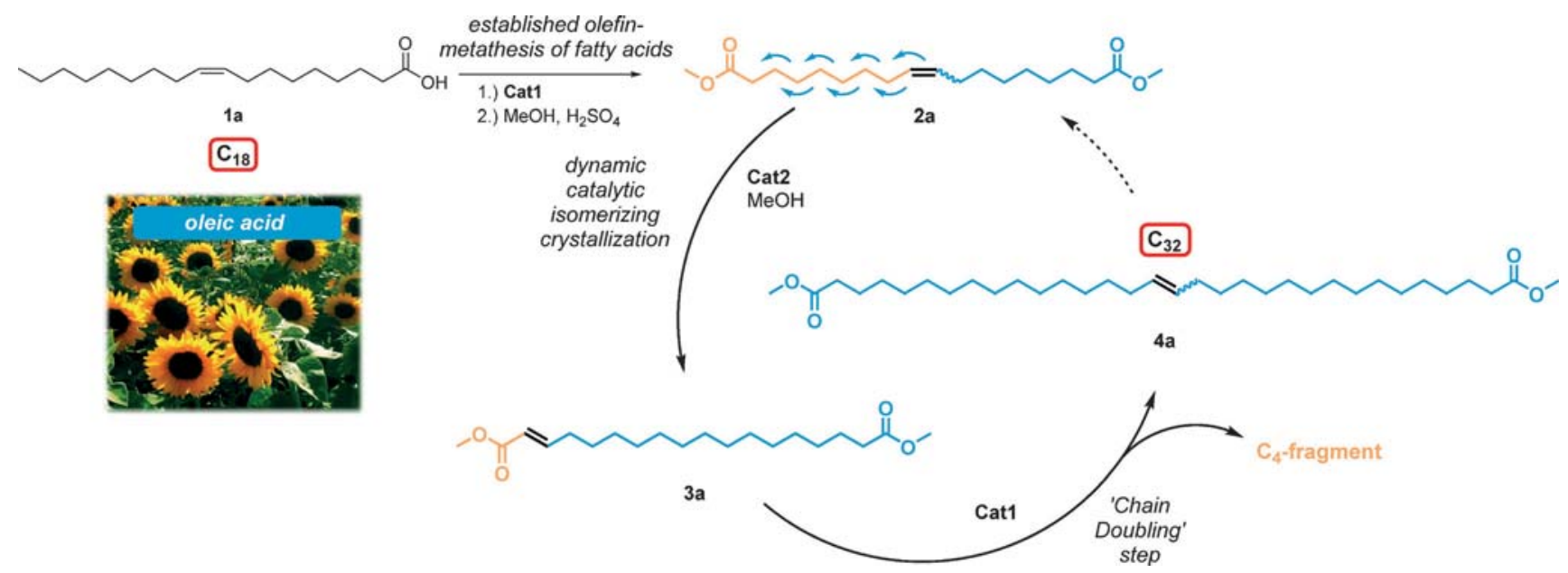

Scheme 1. The "chain doubling" strategy for the preparation of ultra long chain $\alpha, \omega$ diester $4 \mathrm{a}$.

Our concept for chain multiplication relies on a selective shift of the double bond toward a chain end, starting from the above self metathesis product. From here, an olefin meta thesis step will result in a virtual doubling of the chain length, with expulsion of only a small $\mathrm{C}_{4}$ fragment (Scheme 1 ). The internal double bond of the chain doubled products offers itself for hydrogenation for example to generate ultra long chain $\alpha, \omega$ difunctional saturated compounds.

To promote isomerization of the internal double bond of the starting material, we employed $\left[(\mathrm{dtbpx}) \mathrm{Pd}(\mathrm{OTf})_{2}\right]$ (Cat2), bearing the electron rich, bulky diphosphine 1,2 bis (di tert butylphosphino)methyl benzene (dtbpx) ligand, as a highly active catalyst. ${ }^{[24}$ Upon exposure of diester $2 \mathbf{a}$ to a meth anolic solution of $0.5 \mathrm{~mol} \%$ of Cat2, an equilibrium of the isomers of the unsaturated diester differing in the position of the double bond along the chain is attained rapidly. The slightly higher stability of the target $\alpha, \beta$ unsaturated ester $\mathbf{3 a}$ reflects in a slight preference for $\mathbf{3} \mathbf{a}$ in an otherwise statistical isomerization equilibrium. That is, at $30^{\circ} \mathrm{C}$ the $\alpha, \beta$ unsatu rated isomer $\mathbf{3} \mathbf{a}$ is present in $11.7 \%$ with a $(E / Z)$ ratio of 55 (Supporting Information, Figure S1).

To achieve a full conversion into $\mathbf{3} \mathbf{a}$, a physical separation from the continuously catalyzed dynamic equilibrium was employed. From screening of a range of solvents, and temperature sequences, a crystallization from methanol evolved as condition of choice. Owing to its slightly different polarity and lower solubility, crystallization of $\mathbf{3 a}$ occurs preferentially despite its comparatively low concentration in the equilibrium of the double bond iso mers. In combination with continuous replenishment by the ongoing isomeriza tion equilibrium, this provides a net driv ing force for conversion of $\mathbf{2 a}$ into $\mathbf{3} \mathbf{a}$. In this batch type procedure, a decreasing concentration of the double bond isomers in their entirety in solution with increasing amount of already crystallized material is compensated by decreasing the temper ature to maintain supersaturation. In this fashion, a virtually complete yield of chain olefins. crystallized material (95\%) could be obtained for diester 3a. The selectivity for the desired $\alpha, \beta$ unsaturated diester was $84 \%$, as determined by ${ }^{1} \mathrm{H}$ NMR spectroscopy. That is, by the dynamic catalytic isomerizing crystallization procedure the thermodynamic isomer composition can be overcome in favor of the desired $\alpha, \beta$ unsaturated isomer. From the crude products, pure ( $\geq 99 \%)$ 3a was obtained by recrystallization in $70 \%$ overall yield. The remaining internal isomer material can be recycled to the dynamic isomerization/crystalliza tion. ${ }^{[27]}$

With this material in hand, we pursued a "chain doubling" method to the desired ultra long chain structures through olefin metathesis. A direct self metathesis of the $\alpha, \beta$ unsatu rated diesters did not proceed with satisfactory yields, and $\mathbf{4 a}$ was only formed to a minor extent. ${ }^{[28]}$ Apparently, the electron deficient character of the olefinic bond in $\mathbf{3} \mathbf{a}$ reduces its reactivity to such an extent that no significant self metathesis occurs. ${ }^{[29]}$ To overcome this problem, intermittent alkenolysis with electron richer and more reactive short chain olefins was performed (Scheme 2). Readily available, low cost 2 butene or a mixture of internal hexene isomers, respectively, were employed (for further information on alkenolysis of $\mathbf{3} \mathbf{a}$, see the Supporting Information).

To favor a high conversion of $\mathbf{3} \mathbf{a}$, the short chain olefin co reagent was used in excess. In a two step, one pot approach, first the cross metathesis was performed with Cat1, followed by an in situ self metathesis step of the generated monoesters 5a. The latter self metathesis could be induced by simply

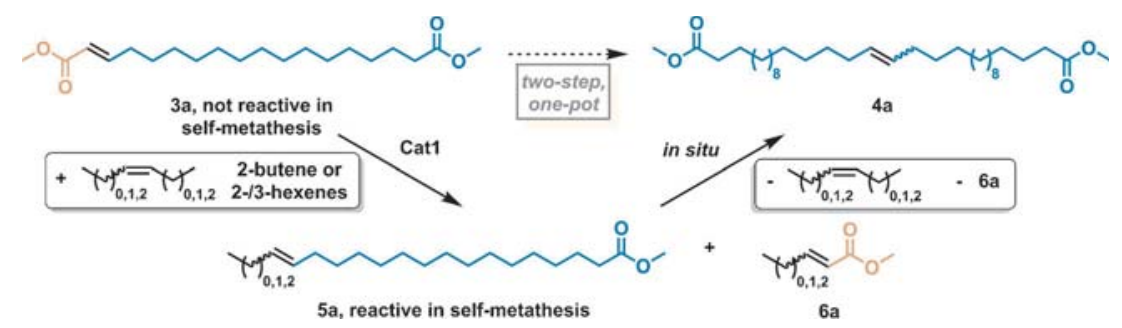

Scheme 2. "Chain doubling" of $\alpha, \beta$ unsaturated diester 3 a via cross metathesis with short 
applying vacuum to the reaction vessel. By this procedure, more than $95 \%$ of the starting material could be transformed into the desired ultra long chain diester $4 \mathbf{a}$.

A potential pitfall in this concept is double bond isomer ization at this stage, a ubiquitous reaction in the presence of ruthenium metathesis catalysts. ${ }^{[30}{ }^{32]}$ This would lead to products of variable chain lengths. To this end, isomerization could be suppressed virtually completely by the choice of appropriate reaction conditions, particularly reaction times and the presence of 1,4 benzoquinone. Hereby, selectivities for the target chain lengths of $\geq 98 \%$ were achieved, as determined by GC analysis (Figure 1).

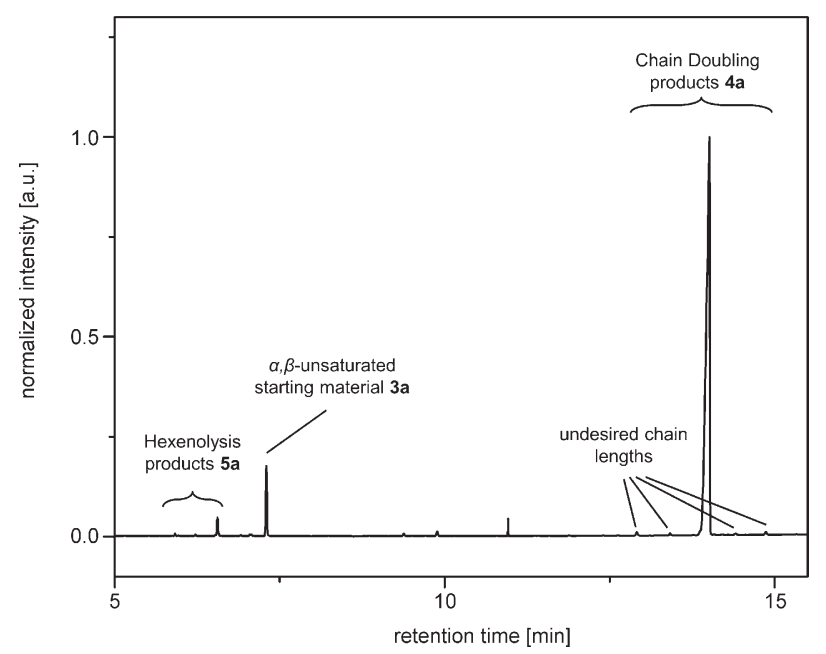

Figure 1. GC trace of crude product after "chain doubling" of $\alpha, \beta$ unsaturated diester 3 a with $2 / 3$ hexenes.
Isolation of the target $\mathrm{C}_{32} \alpha, \omega$ diester was possible by recrystallization to yield an overall $86 \%$ of $\mathbf{4 a}$ in a purity greater than $99 \%$, as determined by GC analysis (Supporting Information, Figure S24).

To access even longer chains, we employed erucic acid (1b) as a starting material. The self metathesis and subse quent esterification in methanol of this $\mathrm{C}_{22}$ unsaturated fatty acid yields an internally unsaturated $\mathrm{C}_{26} \alpha, \omega$ diester. $\mathrm{C}_{26}$ diester $\mathbf{2 b}$ can be used in a double bond isomerization for the preparation of $\alpha, \beta$ unsaturated diester $\mathbf{3 b}$. As the hydro carbon segment of diester $\mathbf{2} \mathbf{b}$ is significantly longer as compared to the segment of diester $\mathbf{2} \mathbf{a}$ from oleic acid, in the isomerization equilibrium the $\alpha, \beta$ unsaturated isomer is present to a lesser extent of $6.7 \%(E / Z=57)$.

As an additional effect of the longer chain, the decreased solubility of the compound has to be overcome in a dynamic isomerizing crystallization. Here, working in ethanol led to a crude yield of $95 \%$ with a selectivity for $\mathbf{3 b}$ of $63 \%$. The pure compound, isolated in $57 \%$ yield after recrystallization, can be chain doubled to $\mathrm{C}_{48} \alpha, \omega$ diester $\mathbf{4 b}$ via the two step, one pot process described above. The desired ultra long chain diester could be obtained in $92 \%$ in a purity greater than $99 \%$, as determined by GC analysis (see the Supporting Information).

As a further demonstration of the utility, reactivity, and purity of the ultra long chain $\alpha, \omega$ diesters, saturated ana logues, corresponding diols and a polyester, were generated (Scheme 3). Saturated diesters $7 \mathbf{a}$ and $\mathbf{7 b}$ were prepared by hydrogenation of $\mathbf{4 a}$ and $\mathbf{4 b}$ using $\mathrm{Pd} / \mathrm{C}$ and hydrogen. The diesters were obtained in 95 and $93 \%$ respectively, in polymerization grade purity as determined by GC (Support ing Information, Figures S26 and S27). Corresponding diols $\mathbf{8} \mathbf{a}$ and $\mathbf{8 b}$ were synthesized by reduction of the ester moieties

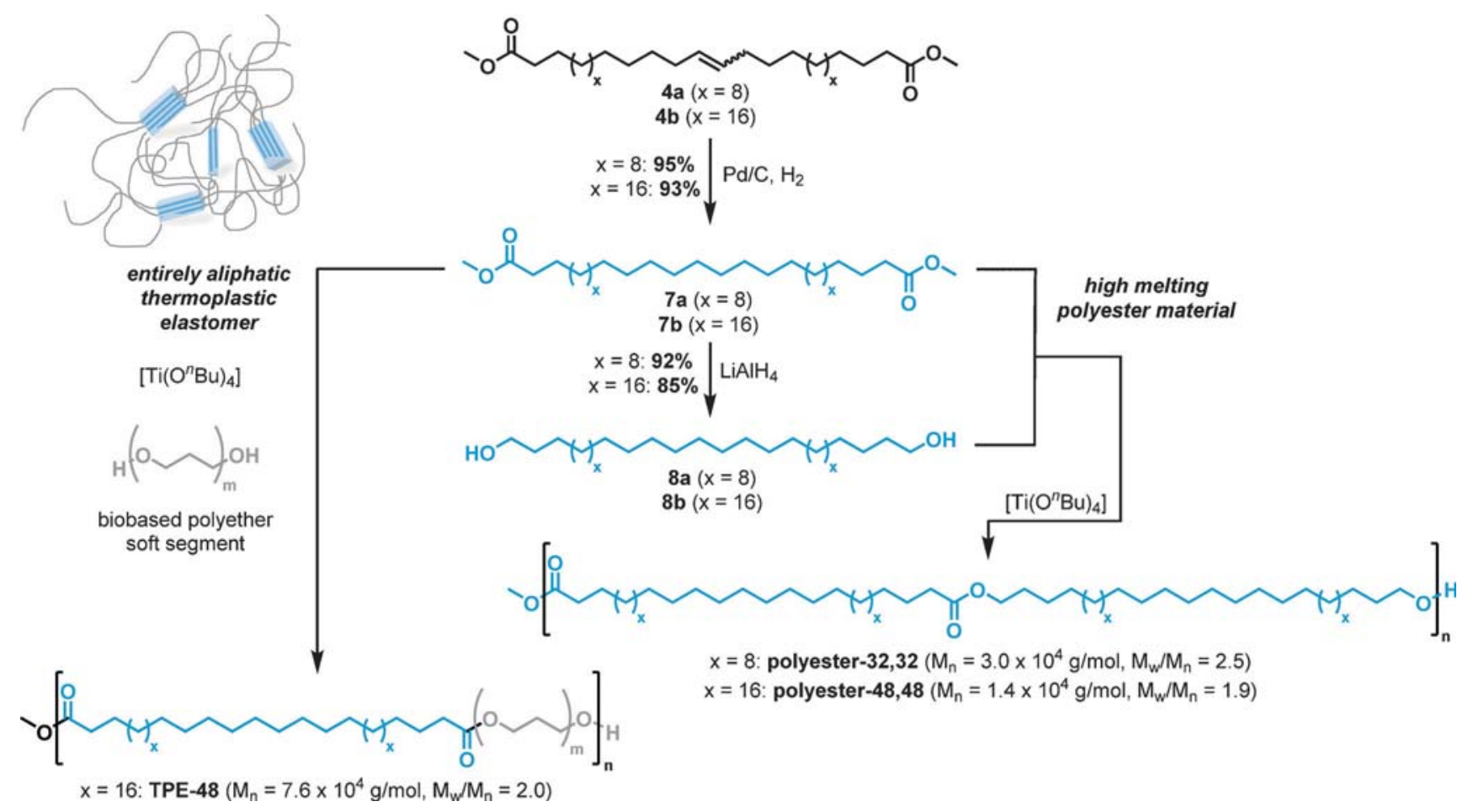

Scheme 3. Preparation of polymeric materials composed of aliphatic ultra long chain monomers. 
using $\mathrm{LiAlH}_{4}$ in $92 \%$ and $85 \%$ yield, respectively. With these compounds accessible in pure form on a multigram scale, we prepared polyesters $\mathbf{3 2 , 3 2}$ and $\mathbf{4 8 , 4 8}$ in a melt polycondensa tion using titanium(IV) tetrabutoxide $\left(\left[\mathrm{Ti}\left(\mathrm{O}^{n} \mathrm{Bu}\right)_{4}\right]\right)$ as a trans esterification catalyst (Supporting Information, Table S3). The influence of the long aliphatic polymethylene segment of the monomers is reflected in the thermal properties of the polyesters and unprecedented melting temperatures were observed. Whereas polyester 32,32 exhibits a peak melting point of $T_{\mathrm{m}}=113^{\circ} \mathrm{C}$, the longer analogue polyester $\mathbf{4 8 , 4 8}$ shows a transition at $T_{\mathrm{m}}=120^{\circ} \mathrm{C}$. Accordingly, polyester $\mathbf{4 8 , 4 8}$ is the first aliphatic polyester that clearly surpasses the melting point of low density polyethylene (LDPE), which is an important benchmark value for many applications. ${ }^{[33]}$

A well crystallizable hydrocarbon segment is also crucial for physical cross linking of aliphatic thermoplastic elasto mers (TPEs). ${ }^{[34]}$ Polycondensation of $\mathrm{C}_{48}$ diester $\mathbf{7 b}$ and poly(trimethyleneglycol) (PPDO, $M_{\mathrm{n}}=3000 \mathrm{~g} \mathrm{~mol}^{-1}$ ) using $\left[\mathrm{Ti}\left(\mathrm{O}^{n} \mathrm{Bu}\right)_{4}\right]$ as transesterification catalyst yielded an elasto meric material (TPE-48, $80 \mathrm{wt} \%$ soft segment) with a narrow melting transition $\left(T_{\mathrm{m}}=80^{\circ} \mathrm{C}\right)$. Tensile tests on injection molded samples revealed a good recovery and strength (Young modulus $E=9 \mathrm{MPa}$ and elongation at break $\varepsilon_{\mathrm{b}}=$ $780 \%$; see the Supporting Information for complete data). By strong contrast, a material based on $\mathrm{C}_{23}$ diester and a correspondingly shorter PPDO as a soft phase with an overall similar composition ( $73 \mathrm{wt} \%$ soft segment) possesses a broad melting transition with a peak at only $T_{\mathrm{m}}=39^{\circ} \mathrm{C}$, which could not even be injection molded on account of the low crystallization temperature and crystallinity. ${ }^{[35]}$ This underlines the ability of the ultra long chain units to function as a crystallizable block in block copolymers. The aforemen tioned mechanical properties of TPE-48 also compare favorably to those of commercial thermoplastic polyester elastomers. $^{[36]}$

This observation of a high crystalline order encourages a study of the self assembly properties of the ultra long chain compounds. To this end, we synthesized $\mathrm{C}_{48}$ dicarboxylic acid 9 with two more hydrophilic carboxylate moieties by sapo nification of diester $\mathbf{7 b}$ with $\mathrm{KOH}$ in THF. Notably, WAXS of 9 as a bulk material already revealed the characteristic orthorhombic polyethylene structure. This differs from the behavior of shorter analogues, as for example $\mathrm{C}_{23}$ dicarboxylic acid $\mathbf{1 0}$ (available from isomerizing alkoxycarbonylation of erucic acid) ${ }^{[37]}$ which pack into a different crystal structure (Figure 2).

To study the self assembly to nanostructures, dicarboxylic acids $\mathbf{9}$ and the $\mathrm{C}_{23}$ analogue $\mathbf{1 0}$ for comparison were dissolved in hot $\mathrm{THF}$ and injected in a $0.01 \mathrm{M}$ aqueous $\mathrm{CsOH}$ solution under ultrasonication. In contrast to $\mathrm{C}_{23}$ diacid $\mathbf{1 0}$, which formed macroscopic precipitates directly upon addition, $\mathrm{C}_{48}$ diacid 9 leads to the formation of a clear transparent dispersion (Figure 2). Dynamic light scattering (DLS) revealed the presence of particle with sizes around $67 \mathrm{~nm}$ with a rather narrow size distribution of PDI $=0.22$.

We believe that the precise ultra long polymethylene sequence acts as a strong driving force for crystallization leading to the formation of crystalline nanoparticles. These particles are stabilized by the hydrophilic interaction of the

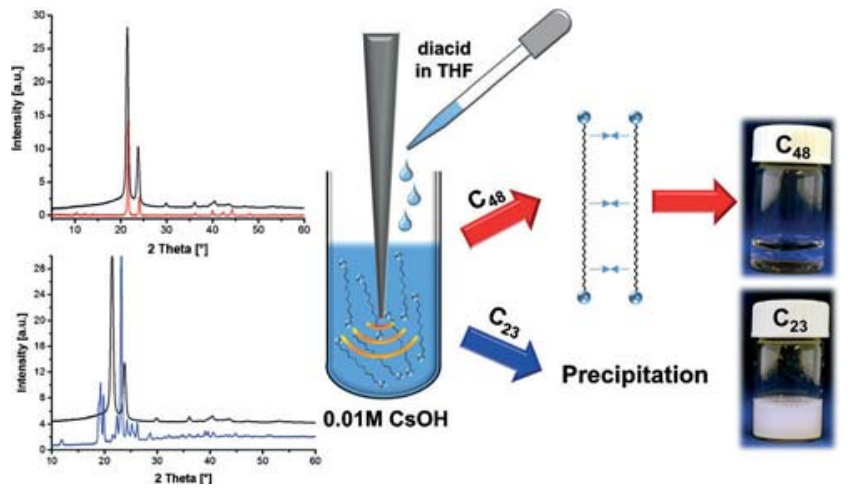

Figure 2. WAXS of semi crystalline polyethylene (black), $\mathrm{C}_{48}$ dicarbox ylic acid 9 (red), and $\mathrm{C}_{23}$ dicarboxylic acid (blue); self assembly behavior of ultra long chain dicarboxylic acid $\mathbf{9}$, and $\mathrm{C}_{23}$ dicarboxylic acid 10 for comparison, in aqueous $\mathrm{CsOH}$ solution.

ordered $\mathrm{COOH}$ groups with the surrounding medium. Astoundingly, transmission electron microscopy (TEM) revealed anisotropic shapes resembling the hexagonal struc ture of typical crystalline polyethylene nanoparticles (Figure 3). ${ }^{[38]}$ The crystal thickness was determined from particles oriented parallel to the electron beam, yielding a value of $6 \mathrm{~nm}$. This thickness corresponds to the length of the fully extended $\mathrm{C}_{48}$ dicarboxylic acid and supports the single layer, extended chain nature of the formed nanocrystals (Figure 3d,e).

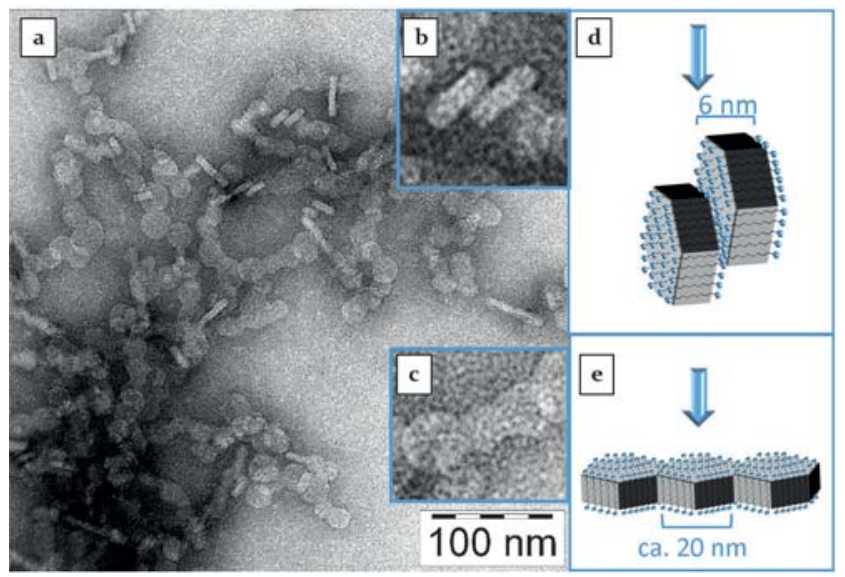

Figure 3. TEM images of stained ( $1 \%$ phosphotungstic acid) $\mathrm{C}_{48}$ nanoparticles: a) overview; section with particles b) parallel and c) per pendicular to the electron beam; d,e) representations of (b) and (c). The blue arrows indicate the direction of the electron beam.

In summary, we show how the methylene chains of two fatty acid molecules can be linked to form an ultra long chain product. This provides access to $\alpha, \omega$ difunctional products with chain lengths that bear potential in many instances, but have not been accessible to date. Readily available unsatu rated fatty acids serve as a starting material. Our approach comprises catalytic steps exclusively, namely olefin isomer ization, crystallization and olefin metathesis, which are all proven to be suited for large scales. ${ }^{[21,39]}$ As the only co 
reagents, short chain olefins which are waste products of the petroleum industry and furthermore can be recycled are required. Almost the entire starting material is incorporated in the product, and the small amount of short chain esters formed can also be useful materials. In principle, the procedure can be iterative as the chain doubled product can serve as the starting material for another cycle of isomer ization/crystallization and metathesis. The potential and unique properties of these new building blocks are high lighted by derived high melting polyesters, thermoplastic elastomeric block copolymers and self assembled nanocrys tals. Particularly the latter two cases reveal the special ability of the ultra long $\mathrm{C}_{48}$ entity to crystallize in a polyethylene like fashion. This clearly differs from shorter analogues, corre sponding to only the length of one fatty acid chain. In this sense, the compounds accessible through the "chain dou bling" approach can be regarded as polyethylene telechel ics $^{[19,40]}$ with an absolutely precise chain length and end group fidelity.

\section{Experimental Section}

General procedure for the synthesis of $\alpha, \beta$ unsaturated diesters $3 \mathbf{a}$ and $\mathbf{3 b}$ : In a typical experiment, a $1 \mathrm{~L}$ round bottom flask with a nitrogen inlet was charged with $25.00 \mathrm{~g}$ of diester $\mathbf{2 a}$ or $\mathbf{2} \mathbf{b}$ that were degassed and subsequently dissolved in $900 \mathrm{~mL}$ of dry alcohol. The solution was cannula transferred into a $1 \mathrm{~L}$ double walled glass reactor with a magnetic stirring bar attached to a cryostat. $0.5 \mathrm{~mol} \%$ of isomerization catalyst Cat 2 dissolved in $5 \mathrm{~mL}$ of the corresponding alcohol were added to the solution, and the reactor was closed and stirred at $250 \mathrm{rpm}$. By means of the cryostat, the reactor was cooled $\left(0.25 \mathrm{~K} \mathrm{~min}^{1}\right)$ to a temperature where the solution became cloudy due to the formation of initial crystals (typical clouding temperature for $2 \mathbf{a}$ : $610^{\circ} \mathrm{C}$; for $\mathbf{2 b}: 2630^{\circ} \mathrm{C}$ ). Subsequently, the reaction was cooled to the final temperature over the course of the designated time (Supporting Information, Figures S2 and S9). Upon reaching this temperature, the isomerization was quenched by the addition of $5 \mathrm{~mL}$ of triethylamine and the slightly yellow precipitate was filtered off. The precipitate was dissolved in a minimal amount of dichloro methane and filtered through a short plug of silica to remove residual catalyst, followed by recrystallization from methanol (in case of $\mathbf{2 a}$ ) or heptane (in case of $\mathbf{2 b}$ ), respectively, yielding the pure diesters $\mathbf{3} \mathbf{a}$ ( $70 \%$ yield) and 3b (57\% yield) in a purity greater than $99 \%$, as determined by ${ }^{1} \mathrm{H}$ NMR spectroscopy and GC analysis.

Dimethyl 1,32 dotricont 16 enedioate (4a): In a typical experi ment, $10.50 \mathrm{~g}(30.8 \mathrm{mmol})$ of $\alpha, \beta$ unsaturated diester 3a were degassed in a Schlenk tube. The diester was suspended in $50 \mathrm{~mL}$ of dry heptane and $39.25 \mathrm{~mL}(26.30 \mathrm{~g}, 312 \mathrm{mmol})$ of $2 / 3$ hexene. $96.6 \mathrm{mg}(154 \mu \mathrm{mol})$ of Cat1 and $33.8 \mathrm{mg}(0.31 \mathrm{mmol})$ of 1,4 benzo quinone were dissolved in $1.0 \mathrm{~mL}$ of dry dichloromethane and added to the mixture via syringe. The mixture immediately turned dark green and was stirred at $40^{\circ} \mathrm{C}$ for $20 \mathrm{~min}$. At $30^{\circ} \mathrm{C}$, the solvent was removed in vacuo, another $50 \mathrm{~mL}$ of dry heptane were added, and the slurry was sonicated for $1 \mathrm{~min}$. Afterwards, the solvent was removed in vacuo, and the mixture was kept at $30^{\circ} \mathrm{C}$ for $15 \mathrm{~min}$ in vacuo. The reaction was quenched by the addition of $10 \mathrm{~mL}$ of ethyl vinyl ether and was stirred at room temperature for $5 \mathrm{~min}$. After addition of $20 \mathrm{~mL}$ of dichloromethane, the reaction was allowed to stir for further $10 \mathrm{~min}$. The dark green solution was filtered through a short silica plug and washed with dichloromethane. The solvent was removed by rotary evaporation, and the colorless solid was dried in vacuo. After recrystallization from methanol and from heptane ultra long chain diester 4a was obtained as colorless crystals in $78 \%(6.47 \mathrm{~g}$,
$12.1 \mathrm{mmol}$ ) yield in a purity greater than $99 \%$, determined by GC analysis.

Dimethyl 1,48 octatetracont 24 enedioate (4b): In a typical experiment, a Schenk flask was charged with $5.00 \mathrm{~g}(11.0 \mathrm{mmol})$ of diester $\mathbf{3 b}$ and degassed. $5 \mathrm{~mL}$ of dry dichloromethane and $13.87 \mathrm{~mL}$ $(9.29 \mathrm{~g}, 110 \mathrm{mmol})$ of $2 / 3$ hexene were added and the mixture was heated to $40^{\circ} \mathrm{C}$. After the diester was dissolved, $34.6 \mathrm{mg}(55 \mu \mathrm{mol})$ of Cat1 and $11.9 \mathrm{mg}(110 \mu \mathrm{mol})$ of 1,4 benzoquinone were added in $0.5 \mathrm{~mL}$ of dry dichloromethane. The solution was stirred for $15 \mathrm{~min}$ at $40^{\circ} \mathrm{C}$, cooled down to room temperature, and $10 \mathrm{~mL}$ of dry heptane were added. Subsequently the volatiles were removed slowly in vacuo. The resulting solid was dissolved in dichloromethane and ethyl vinyl ether was added to quench the reaction. After stirring for $15 \mathrm{~min}$ at room temperature the solution was diluted with dichloro methane and filtered through a short silica plug to remove residual catalyst. After recrystallization from heptane, ultra long chain diester 4b was obtained as colorless crystals in $92 \%$ yield in a purity greater than $99 \%$, determined by GC analysis.

\section{Acknowledgements}

We gratefully acknowledge financial support by the Stiftung Baden Württemberg. We thank Lars Bolk for GPC and DSC measurements and Dr. Marina Krumova for TEM measure ments. Support by the DFG (SFB1214) is acknowledged.

\section{Conflict of interest}

The authors declare no conflict of interest.

Keywords: fatty acids - olefin metathesis - polyethylene . double bond isomerization $\cdot \alpha, \omega$ difunctional units

[1] F. C. Naughton, J. Am. Oil Chem. Soc. 1974, 51, 65.

[2] A. Künkel, J. Becker, L. Börger, J. Hamprecht, S. Koltzenburg, R. Loos, M. B. Schick, K. Schlegel, C. Sinkel, G. Skupin, M. Yamamoto, in Ullmann's Encyclopedia of Industrial Chemistry, Wiley VCH, Weinheim, 2016.

[3] U. Biermann, U. Bornscheuer, M. A. R. Meier, J. O. Metzger, H. J. Schäfer, Angew. Chem. Int. Ed. 2011, 50, 3854; Angew. Chem. 2011, 123, 3938.

[4] F. Stempfle, P. Ortmann, S. Mecking, Chem. Rev. 2016, 116, 4597. [5] A. Weerheim, M. Ponec, Arch. Dermatol. Res. 2001, 293, 191.

[6] J. van Smeden, M. Janssens, G. S. Gooris, J. A. Bouwstra, Biochim. Biophys. Acta Mol. Cell. Biol. Lipids 2014, 1841, 295.

[7] P. Blokker, S. Schouten, H. van den Ende, J. W. de Leeuw, P. G. Hatcher, J. S. Sinninghe Damsté, Org. Geochem. 1998, 29, 1453.

[8] F. Gelin, I. Boogers, A. A. M. Noordeloos, J. S. S. Damsté, P. G. Hatcher, J. W. De Leeuw, Geochim. Cosmochim. Acta 1996, 60, 1275.

[9] P. Blokker, S. Schouten, H. van den Ende, J. W. De Leeuw, J. S. Sinninghe Damsté, Phytochemistry 1998, 49, 691.

[10] F. Gelin, I. Boogers, A. A. M. Noordeloos, J. S. S. Damste, R. Riegman, J. W. De Leeuw, Org. Geochem. 1997, 26, 659.

[11] O. Bertheas, P. Metzger, C. Largeau, Phytochemistry 1999, 50, 85.

[12] P. E. Kolattukudy, Science 1980, 208, 990.

[13] K. E. Espelie, P. E. Kolattukudy, Plant Physiol. 1979, 63, 433.

[14] G. Eglinton, D. H. Hunneman, K. Douraghi Zadeh, Tetrahedron 1968, 24, 5929.

[15] B. A. Lewis, D. M. Engelman, J. Mol. Biol. 1983, 166, 211. 
[16] E. E. Rusanova, Y. L. Sebyakin, L. V. Volkova, R. P. Evstigneeva, J. Org. Chem. USSR 1984, 20, 248.

[17] K. E. Augustin, H. J. Schäfer, Liebigs Ann. Chem. 1991, 1037.

[18] H. Makio, T. Ochiai, J. i. Mohri, K. Takeda, T. Shimazaki, Y. Usui, S. Matsuura, T. Fujita, J. Am. Chem. Soc. 2013, 135, 8177.

[19] I. German, W. Kelhifi, S. Norsic, C. Boisson, F. D'Agosto, Angew. Chem. Int. Ed. 2013, 52, 3438; Angew. Chem. 2013, 125, 3522.

[20] Z. Jian, L. Falivene, G. Boffa, S. O. Sánchez, L. Caporaso, A. Grassi, S. Mecking, Angew. Chem. Int. Ed. 2016, 55, 14378; Angew. Chem. 2016, 128, 14590.

[21] S. Chikkali, S. Mecking, Angew. Chem. Int. Ed. 2012, 51, 5802; Angew. Chem. 2012, 124, 5902.

[22] H. L. Ngo, K. Jones, T. A. Foglia, J. Am. Oil Chem. Soc. 2006, 83, 629.

[23] H. Mutlu, R. Hofsa, R. E. Montenegro, M. A. R. Meier, RSC Adv. 2013, 3, 4927.

[24] G. Walther, J. Deutsch, A. Martin, F. E. Baumann, D. Fridag, R. Franke, A. Köckritz, ChemSusChem 2011, 4, 1052.

[25] P. Roesle, C. J. Dürr, H. M. Möller, L. Cavallo, L. Caporaso, S. Mecking, J. Am. Chem. Soc. 2012, 134, 17696.

[26] P. Roesle, L. Caporaso, M. Schnitte, V. Goldbach, L. Cavallo, S. Mecking, J. Am. Chem. Soc. 2014, 136, 16871.

[27] After removing catalyst residues by filtration over silica, a second isomerization can be conducted upon addition of fresh catalyst. This step can be performed without a significant loss in activity and selectivity and a crude yield of $84 \%$ can be obtained with a selectivity for the desired diester $3 \mathbf{a}$ of $83 \%$. Note that this apparently somewhat lower crude yield from the recycled isomers may be due to a higher concentration of residual methyl oleate in the filtrate used as starting material for a second cycle of isomerizing crystallization.

[28] Note that performing a self metathesis of $\alpha, \beta$ unsaturated diester 3a under reduced pressure at elevated temperatures resulted in isomerization of the double bond, leading to a range of different chain lengths in the self metathesis products with incomplete conversion of the starting material.

[29] A. K. Chatterjee, T. L. Choi, D. P. Sanders, R. H. Grubbs, J. Am. Chem. Soc. 2003, 125, 11360.

[30] S. E. Lehman, Jr., J. E. Schwendeman, P. M. O'Donnell, K. B. Wagener, Inorg. Chim. Acta 2003, 345, 190.

[31] S. H. Hong, M. W. Day, R. H. Grubbs, J. Am. Chem. Soc. 2004, 126, 7414.

[32] S. H. Hong, D. P. Sanders, C. W. Lee, R. H. Grubbs, J. Am. Chem. Soc. 2005, 127, 17160.

[33] D. Jeremic, in Ullmann's Encyclopedia of Industrial Chemistry, 7th Ed., Wiley VCH, Weinheim, 2014.

[34] K. S. O'Connor, A. Watts, T. Vaidya, A. M. LaPointe, M. A. Hillmyer, G. W. Coates, Macromolecules 2016, 49, 6743.

[35] F. Stempfle, B. Schemmer, A. L. Oechsle, S. Mecking, Polym. Chem. 2015, 6, 7133.

[36] T. Ouhadi, S. Abdou Saber, H. G. Wussow, L. M. Ryan, L. Plummer, F. E. Baumann, J. Lohmar, H. F. Vermeire, F. L. G. Malet in Ullmann's Encyclopedia of Industrial Chemistry, 7th Ed., Wiley VCH, Weinheim, 2014.

[37] F. Stempfle, D. Quinzler, I. Heckler, S. Mecking, Macromole cules 2011, 44, 4159.

[38] C. H. M. Weber, A. Chiche, G. Krausch, S. Rosenfeldt, M. Ballauff, L. Harnau, I. Göttker Schnetmann, Q. Tong, S. Meck ing, Nano Lett. 2007, 7, 2024.

[39] A. Behr, in Ullmann's Encyclopedia of Industrial Chemistry, 6th Ed., Wiley VCH, Weinheim, 2010.

[40] L. M. Pitet, M. A. Hillmyer, Macromolecules 2011, 44, 2378. 